\title{
Poetische Okkasionalismen bei Nestroy und Handke: ein literarisch-linguistischer und korpuslinguistischer Kontrast
}

\author{
Wolfgang U. Dressler \\ Austrian Academy of Sci- \\ ences \\ Postgasse 7 \\ 1010 Vienna - Austria \\ wolf- \\ gang. dresser@uni- \\ vie.ac.at
}

\author{
Vanessa Hannesschläger \\ Austrian Academy of Sci- \\ ences \\ Sonnenfelsgasse 19 \\ 1010 Vienna - Austria \\ vanessa.han- \\ nesschlae- \\ ger@oeaw.ac.at
}

\author{
Barbara Tumfart \\ Austrian Academy of Sci- \\ ences \\ Sonnenfelsgasse 19 \\ 1010 Vienna - Austria \\ barbara.tum- \\ fart@oeaw.ac.at
}

\begin{abstract}
In diesem Beitrag wollen wir sprachliche Neubildungen (präziser: Okkasionalismen) als Elemente poetischer Kreativität bei Johann Nepomuk Nestroy und Peter Handke mit korpuslinguistischen Methoden in neuer Weise untersuchen und vergleichen, zeigen, was mit diesen zum ersten Mal möglich ist und damit auch der Literatur- und Theaterwissenschaft neue Möglichkeiten eröffnen.
\end{abstract}

\section{$1 \quad$ Einleitung}

Computerunterstützte Methoden ermöglichen und fördern in der Kombination qualitativer und quantitativer Arbeitsansätze neue Fragestellungen für linguistische und literaturkritische Forschung. Natürlich wurden Korpora bereits vor der Verbreitung digitaler Medien in vielen Bereichen der Linguistik verwendet. „In der Korpuslinguistik sollen Erkenntnisse gewonnen werden, die über die beobachtete Datenmenge hinaus generalisierbar sind und so unsere Einsicht in die Funktion und Verwendung einer Sprache vertiefen“ (Lemnitzer/Zinsmeister 2006: 52). Durch die computertechnische Aufbereitung, Speicherung und Weiterverarbeitung dieser Daten bieten sich aber nun mehr Möglichkeiten, sprachliche Strukturen, sprachliche Regularitäten oder Irregularitäten nachvollziehbar und belegbar zu erforschen, zu analysieren und sie in der Folge auch weiteren Forschungsdisziplinen, wie beispielsweise der literaturwissenschaftlichen Analyse und Interpretation zur Verfügung zu stellen (vgl. Storrer 2011). Lüdeling/Zeldes (2009) illustrieren die unterschiedlichen Arbeitsweisen der Disziplinen "copus linguistics", "literary computing" und "computational linguistics" und plädieren für einen wechselseitigen Austausch der gewonnenen Ergebnisse, der Forschungsfragen wie auch für einen je nach Anfrage angepassten Einsatz der verschiedenen Tools, Abfrage- und Analysemechanismen. "Where in the past computational linguists may have used a linguistic corpus to create taggers and parsers, and linguists in turn used these tools on corpora digitized by the digital humanities, we are entering a stage where work using the same resource is becoming possible on both an interdisciplinary level and an interpersonal level, between researchers working separately" (Lüdeling/Zeldes 2009: 170). Durch den Einsatz korpuslinguistischer Methoden und hierbei besonders durch die Kontrastierung eines speziellen Autorencorpus mit größeren zeitgenössischen, aber auch mit zeitlich jüngeren Textsammlungen, können belegbare Aussagen über den spezifischen Sprachgebrauch eines Autors gemacht werden. Als weiterer Schritt können diese durch corpuslinguistische Fragestellungen gewonnenen Ergebnisse einer weiteren literaturwissenschaftlichen Analyse unterzogen werden. Unter Einbeziehung ko(n)textueller Positionierung von spezifischen sprachlichen Phänomenen können beispielsweise Aussagen über deren Funktionen in den einzelnen Textpassagen bzw. im Gesamtkorpus eines Autors getätigt werden.

Im vorliegenden Beitrag wurden bewusst zwei Autoren gewählt, die zeitlich weit voneinander entfernt sind und in ihrem literarischen Schaffen unterschiedlichen Genres zuzuordnen sind. Gemeint sind Johann Nepomuk Nestroy (1801-1862) und Peter Handke (geboren 1942): Nestroy darf als der berühm- 
teste österreichische Komödiendichter bezeichnet werden. Während auch Handke zwar dem Bühnengenre viele Arbeiten gewidmet hat, ist sein Prosawerk doch wesentlich umfangreicher und wird deshalb in dieser Arbeit Gegenstand der Untersuchung sein - auch, um einen noch deutlicheren Kontrast zwischen den Untersuchungsgegenständen für die Analyse fruchtbar zu machen. Den beiden Autoren ist allerdings ihre Besonderheit innerhalb der deutschsprachigen bzw. österreichischen Literatur gemein, die sich durch ein besonders großes und umfangreiches Werk, durch einen spezifischen und gezielt eingesetzten Sprachgebrauch, ein besonderes Sprachbewusstsein und durch eine unverminderte Präsenz in der kulturellen Wahrnehmung manifestiert.

\section{Okkasionalismen}

Okkasionalismen sind ein besonderer Typ von Hapax Legomena, die zumindest seit Aristophanes (vgl. Peppler 1902) ein Mittel poetischer Kreativität sind (Murat 2015). Okkasionalismen sind Wortneubildungen, welche Autoren für eine einmalige Verwendung an einer bestimmten Textstelle kreieren und die nicht dazu bestimmt sind in der Sprachgemeinschaft als Neologismen anerkannt und wieder verwendet zu werden, es sei denn als Zitate (vgl. Christofidou 1994). Auch verwendet sie ein Autor kein zweites Mal, außer wenn er damit eine im Text wiederkehrende Person charakterisiert. Der Terminus geht auf Chanpira (1966) zurück, der damit poetische Neubildungen von Neologismen unterscheiden wollte. Okkasionalismen verletzen die lexikalische Norm, die Spachverwendung auf existierende von der Sprachgemeinschaft anerkannte Wörter zu beschränken. Solange eine Wortneubildung aber mit Hilfe produktiver Regeln erfolgt, also der Norm der Wortbildung entspricht, neue Wörter nur mit Hilfe produktiver Wortbildungsregeln zu bilden, ist dies eine dem normalen Sprachgebrauch entsprechende poetische Lizenz. Wie Dressler (1981, 2007) argumentiert hat, ist die poetische Lizenz kühner, wenn Okkasionalismen mit Hilfe unproduktiver Wortbildungsregeln gebildet werden, wie z.B. Tod und Aber+tod (Herrmann Broch: Der Tod des Vergil), und besonders, wenn sie ungrammatisch gebildet sind, wie z.B. Hautevole +er+er (Nestroy: Lady und Schneider). Diese Skala der poetischen Lizenz betrifft die paradigmatische Achse der Sprache. Aber auch die syntagmatische Achse der Sprache ist wichtig, nämlich wie Okkasionalismen in den Kotext eingebettet sind (vgl. Dressler \& Mörth 2012).

Wir untersuchen und kontrastieren also Okkasionalismen bei Nestroy und Handke, bei diesem auch seine häufig gebildeten okkasionalistischen Gappingkonstruktionen, welche bei Nestroy fast überhaupt nicht vorkommen.

Die Prozedur der Analyse von poetischen Okkasionalismen, die wir hier mit Beispielen aus Nestroy exemplifizieren, beginnt mit der Introspektion, dass einem bei der Lektüre ein Wort unbekannt erscheint und damit ein Kandidat für die Identifikation als poetischer Okkasionalismus wird. Diese Annahme muss aber überprüft werden, was vor der Existenz umfangreicher elektronischer Korpora nur in geringem Maße möglich war. So hat Hunger (1999: 89) Purzler (zweimal im Mädl aus der Vorstadt III 11) als Nestroysche Neubildung bezeichnet, in einem elektronischen Korpus kommt es aber zweimal vor. Zunächst muss festgestellt werden, ob ein angenommener Okkasionalismus tatsächlich nur ein einziges Mal bei einem Autor vorkommt, was an den elektronischen Korpora der ÖAW von Nestroy und Handke überprüft wird. Dann muss die elektronische Suche auf Korpora derselben Zeitperiode ausgeweitet werden und schließlich auch auf Korpora späterer Zeitabschnitte, denn ein Vorkommen in späteren Perioden kann auf früheren, elektronisch nicht erfassten Sprachgebrauch zurückgehen. Deshalb sind Wörter, die nach Nestroy mit vielen Belegen auftauchen, als Okkasionalismen Nestroys auszuschließen. Ein Beispiel ist normal+mäßig, welches bei Nestroy nur einmal vorkommt (zweimal im Mädl aus der Vorstadt II 7), aber in einem elektronischen Korpus der ÖAW 57 Belege hat.

Bei Einzelauftreten anderswo ist eine qualitative Beurteilung notwendig. Klar ist der Fall des Nestroy'schen Okkasionalismus Eh'stand + s+lotterie (im Gewürzkrämerkleeblatt von 1845), welches im Titel von G. Köberles Lustspiel Ehestandslotterie (von 1853) wiederkehrt: Hier hat offensichtlich Nestroys Rivale von diesem abgeschrieben. Interessanter ist der Fall der Nominalphrase mit unsrer Dräng+er Schar (Mädl aus der Vorstadt II 12), welche wortwörtlich in einem Arbeiterlied und bei Ferdinand Lassalle wiederkehrt. In diesen wurde offenbar, dass der in der Arbeiterbewegung wegen seines einfühlsamen Eingehens auf einfache Leute und seiner häufigen antikapitalistischen Kritik beliebte Nestroy zitiert. 


\section{Johann Nestroy}

Johann Nepomuk Nestroy (1801-1862) gilt unbestritten als einer der Hauptvertreter des deutschsprachigen Volkstheaters und als einer der bedeutendsten Komödiendichter der österreichischen Theaterlandschaft des 19. Jahrhunderts. Karl Kraus, der selbst als „Sprachgläubiger“ charakterisiert werden kann, bezeichnete Nestroy anlässlich dessen 50. Todestages als ,erste[n] deutschen[n] Satiriker, in dem sich die Sprache Gedanken macht über die Dinge“ (Weiss 1995: 201). Friedrich Sengle (1980: 203) porträtiert Nestroy als „Sprachkünstler“, bei dem die komische Sprache ,aktiv und üppig wucher[t]“ und geht sogar so weit, von einer „Sprachbesessenheit“, einer ,ganz originale[n] Sprachkraft“ (Sengle 1980: 206) zu sprechen. Auch die neuere Nestroy-Forschung (Hein 1970, 1990) betont die sprachliche Ausnahmeerscheinung seiner Volksstücke und sieht Nestroys satirische Kunst und die Erfolge seiner Bühnenwerke in seiner Sprachkunst begründet. Spiel und Satire bilden quasi das „Kräfteparallelogramm“ (Hein 1970: 159) der Komödie und nehmen damit eine Doppelfunktion bei Nestroy ein. Da Nestroys Stücke vor allem aufgrund seines speziellen Sprachbewusstseins unverändert zeitaktuell erscheinen (und regelmäBig neu inszeniert werden), erscheint eine Untersuchung der Okkasionalismen von besonderer Bedeutung. Eine kritische Gegenüberstellung zu der zeitgenössischen Theaterproduktion, wie auch des modernen Sprachgebrauchs ist hierfür genauso unverzichtbar wie eine Intensivierung der corpuslinguistischen Untersuchungsweisen. Durch diese Arbeitsweise können bisherige Analysen zu Nestroys Sprachgebrauch (Gengnagel 1962, Panagl 1983, Hunger 1999) mit neuen Ergebnissen und Antworten auf bislang nicht mögliche Fragestellungen erweitert werden.

Es wurden Okkasionalismen in folgenden drei Theaterstiucken Nestroys aus den 1840er Jahren untersucht: Das Mädl aus der Vorstadt oder Ehrlich währt am längsten (Posse mit Gesang in 3 Akten, 1841), Der Zerrissene (Posse mit Gesang in 3 Akten, 1844) und Das Gewürzkrämer-Kleeblatt (Posse mit Gesang in 3 Aufzügen, 1845), im Folgenden abgekürzt als Mädl, Zerriss, Gewürz. Das Viennese Theatre Corpus (VTC), das Austrian Academy Corpus (AAC), das Austrian Media Corpus (AMC) und das Deutsche Textarchiv (DTA) dienten zum Abgleich der bei Nestroy auffälligen Wortneubildungen. Das VTC der Academiae Corpora (AC) der Österreichischen Akademie der Wissenschaften (ÖAW) umfasst Theaterstücke von Zeitgenossen im Zeitraum von etwa 1840 bis 1870 und weist eine Tokenanzahl von etwa 750.000 auf. Das AAC der ÖAW deckt unterschiedlichste Textsorten aus dem Zeitraum von 1848 bis zur ersten Hälfte des 20. Jahrhunderts ab und besteht aus etwa 500 Millionen Token. Das AMC der ÖAW ist ein Corpus österreichischer Printmedien ab den 1990er Jahren mit einem Umfang von über 8 Milliarden Token. Das DTA mit einer Anzahl von ca. 100 Millionen running words ermöglicht durch die Volltextsuche in Texten aus dem 17. und 18. Jahrhundert Vorkommen von Wörtern in der Periode vor Nestroy zu untersuchen.

\section{Sind Nestroys Okkasionalismen Nachbildungen aus dem Französischen?}

Die österreichische Theaterliteratur zeichnet sich im gesamten 19. Jahrhundert durch eine große Abhängigkeit von der nachbarschaftlichen Produktion aus, wobei neben der englischen Theaterproduktion das französische Theater eine fast monopolartige Stellung inne hatte (vgl. Bachleitner 1989). Generell wurden innerhalb dieses gängigen Prozesses des Kulturtransfers die französischen (und englischsprachigen) Stücke in ein deutsches Wirkungsfeld quasi verpflanzt (vgl. Ruprecht 1965, Tumfart 2012, 2018). Yates (2008: 44) geht sogar so weit, von dem Werk zahlreicher französischer Lustspielautoren als ,domesticated in Vienna“" zu sprechen. Nestroys Werk betreffend, kann man in Summe mehr als 30 seiner Possen auf französische Dramen, Komödien, Opern und Romane zurückführen. Bei seiner Art der Übersetzung bzw. Bearbeitung kann man im Vergleich zu seinen Zeitgenossen von einer „creative adaptation“ (Yates 1972: 120) sprechen. Er übernimmt zwar Grundzüge der Handlung und den Großteil der Figuren, erschafft allerdings in einer kritischen Distanz zur Vorlage Dialoge und Monologe neu (vgl. Doering 1994), in dem er diese verknappt, den Sprachduktus erhöht und durch Witz und pointierte Rede- und Gegenrede ,sprunghafte Assoziationen provoziert“ (Fischer 1962: 191).

Für die drei für diese Untersuchung ausgewählten Nestroystücke diente ebenfalls jeweils ein französisches Theaterstück als Vorlage. Für Mädl ist die Vorlage La Jolie Fille du faubourg (ComédieVaudeville, 1840) von De Kock et Varin, für Zerriss L'Homme blasé (Comédie-Vaudeville, 1843) von Duvert und Lauzanne und für Gewürz fungierte Trois Épiciers (Vaudeville, 1840) von Lockroy und Anicet-Bourgeois als Vorlage. Die französischen Originale werden im Folgenden als blasé, Joli und 
épiciers abgekürzt und können in der historisch-kritischen Nestroy-Ausgabe der internationalen Nestroy-Gesellschaft nachgelesen werden.

Hier ist zu klären, ob Nestroys Okkasionalismen tatsächlich originelle Kreationen oder - wenigstens teilweise - Übertragungen aus französischen Okkasionalismen sind. Unsere Analyse hat ergeben, dass die französischen Vorlagen der drei untersuchten Nestroystücke überhaupt keine Okkasionalismen enthalten. Auch übersetzt Nestroy fast nie französische Phrasen in deutsche Neubildungen, gleichgültig ob Komposita oder Derivationen. Eine Ausnahme bildet die produktive verbale Präfigierung im Partizipium von in der an+geverselten Landnatur (Zerriss II 9), welches eine sehr freie Übertragung aus l'affreuse bucolique (blasé II 5) ist, wobei sich die Denotation von affreux, scheußlich' in der negativen Konnotation des verbalen Diminutivs von an+geverselt wieder findet. In Mädl II 15 hat Nestroy coquett-erie der Vorlage (Joli III 1) durch ungrammatisches Kokett+ur ersetzt, d.h. die einfache Übernahme durch das Fremdwort Koketterie vermieden. In Gewürz (II 4) ersetzt Nestroy des femmes qu'on enferme (épiciers II 4) durch das synthetische Phrasenkompositum das Weibereinsperren, in III 5 das existierende Kompositum guet-apens ,Hinterhalt‘ (III 4) durch das bedeutungsmäßig sehr verschiedene Kompositum Damenfopperei.

Fast alle anderen Okkasionalismen Nestroys finden sich nicht in Übertragungen aus den französischen Vorlagen, sondern in seinen Zusätzen, besonders in seinen Räsonnements und Dialogen, aber fast nicht in Liedern. Nestroys Kreativität und poetische Zielsetzung zeigt sich in Relation zu seinen Vorlagen in der Neuschaffung sprachlicher Formulierungen, Ergänzungen und Abweichungen und gesteigerter Qualität der Dialoge, die mitunter einer parodistischen Umgestaltung gleichkommen (vgl. Fischer 1962, Panagl 2003).

\section{Grad der Kühnheit von Nestroys poetischer Lizenz}

Dressler $(1981,2007)$ hatte angenommen, dass sich Nestroys Okkasionalismen durch hochgradige Kühnheit auszeichnen und dafür nach der Skala von § 2 Beispiele ungrammatischer Wortbildungen und von Neubildungen nach dem Muster unproduktiver (also fossiler) Wortbildungsregeln angeführt (vgl. auch Hunger 1999 und Panagl 1983). Aber bei genauer Analyse der Okkasionalismen in den drei untersuchten Theaterstücken hat sich ergeben, dass von 103 Okkasionalismen nur ein einziger nach einem unproduktiven Muster gebildet ist, nämlich zorn+erbleichen in einem Lied (Mädl II 12). Ungrammatisch sind nur die bereits genannte Kokett+ur (Mädl II 15) und Alle+tag+s+genüsse (Zerriss I 5) mit ungrammatischem Ersatz des Pluralsuffixes $-e$ durch das Interfix $-s-$. Strittig ist schließlich ver+unter+halten (Zerriss I 3), welches wir im Gegensatz zu Panagl (1983) und Hunger (1999) nicht für ungrammatisch, sondern nur für ungewöhnlich halten, weil das Präfix ver-gewöhnlich nur vor einsilbigen Präfixen auftritt (ver+un+reinigen, ver+ab+reichen, ver+aus+lagen usw.). Außerhalb unser Skala steht die extragrammatische, durch Kontamination gebildete okkasionalistische Wortneubildung Amour+ett+el aus den Fremdwörtern Amour (frz.) und Amor+ette (It.), deriviert mit dem im 19. Jahrhundert noch produktiven Diminutivsuffix $-(e) l$.

Alle anderen 99 (bzw. mit Amour+ett+el 100) Okkasionalismen sind aber mit Hilfe produktiver Wortbildungsregeln gebildet, und zwar 62 Komposita und 37 Derivationen. Mit diesem klaren Überwiegen von Kompositionen entspricht Nestroy dem Grundcharakter der deutschen Wortbildung, stärker kompositionell als derivationell zu sein (vgl. Gaeta/Schlücker 2012).

Die nach produktiven Mustern gebildeten Komposita sind sowohl interfixlose, rein konkatenative wie Podagra+füß ' (Mädl I 6), interfigierte wie ebendort Kapitalist+en+gefühl, synthetische Komposita wie Glück+zerstörer (Zerriss I 17), Phrasenkomposita wie Spanische+Rohr+Rache (Zerriss II 11), schnee + blüh + wei $\beta+$ ge + wasch $+n ' e$ (Zerriss 14 ), suffigierte Phrasenkomposita wie Hinter + die $+O h$ ren+hab+igkeit (Gewürz II 4).

Die nach produktiven Mustern gebildeten Derivationen sind von einer großen Vielfalt. Mehr als einmal kommen vor: deverbale Ereignisbildungen wie der juristische Hums+er (Zerriss I 9), nomina actionis wie Plantier+ung ( $M a ̈ d l$ I 3), damals noch produktive nominale Diminutive wie Bräutigamm+l (Zerriss III 3), verbale Diminutive wie othell+erl+n (Gewürz III 9), verbale Präfigierung wie herab+gurgeln (Mädl II 12), denominale Adjektive wie nix Fiakr+isches (Zerriss I 6), Umwandlung von Partizipien in Adjektive wie ein rangier-ter+er Mann (Mädl II 5).

Nestroy ist also nicht auf der paradigmatischen Achse der verwendeten Wortbildungsregeln besonders kühn, wohl aber auf der bisher noch nicht untersuchten syntagmatischen Achse der Zusammensetzung 
der Teile, was am besten bei der Nominalkomposition zu beobachten ist. Nestroys Kühnheit der Wortneubildung zeigt sich hier in zwei Aspekten:

Erstens bildet er Komposita, deren Konstituenten eine geringe Familiengröße haben. Normalerweise werden neue Komposita aus Konstituenten gebildet, die eine große Familiengröße aufweisen, d.h. häufig in derselben Position (als Erst- oder Zweitglied) bereits in vielen Komposita vorkommen (vgl. Hay/Baayen 2002, Mulder et al. 2014). Ein Okkasionalismus mit geringer Familiengröße der Konstituenten z.B. Mathilden+schnipfer (Zerriss I 4). Ein Vorname als Erstglied und besonders Mathilde ist rar. Ein Zweitglied Schnipfer haben wir nur in pejorativem Haupt+schnipfer (zweimal bei Nestroy), Äpfel+schnipfer, einem Spottnamen des bayrischen Königs Ludwig I, Streu+schnipfer (Ganghofer) und im Schimpfwort Regiments+schnipfer gefunden. Ein Beispiel, wo nur das Zweitglied ungewöhnlich ist, ist Drauf+kommung (Gewürz II 23), für dessen Zweitglied wir als analoges Kompositum nur Frei+bekommung, mit einem ähnlichen Zweitglied gefunden haben, wobei aber kommen ein intransitives, bekommen ein transitives Verb ist.

Zweitens bildet Nestroy semantisch überraschende Komposita, wie z.B. Liebes+mathematiker ( $M \ddot{d} d l$ II 15), welches auf eine ungewöhnliche quasimathematische Operation hinweist, während vergleichbare Kalkulationen durch einen Urlaubs+mathematiker oder Pensions+mathematiker immerhin genaue Zählungen implizieren. Alle anderen belegten Komposita wie Versicherungs+mathematiker, Spitzen+mathematiker und sehr viele andere sind alle Fachmathematiker. Es gibt im 19. Jahrhundert auch keine vergleichbaren Komposita mit Erstglied Liebe(s). Liebes+mathematik und Liebes+geometrie haben wir erst im 21. Jahrhundert im AMC gefunden.

Bei den durch Gapping verbundenen Okkasionalismen, die bei Nestroy selten vorkommen, wie Blumen- und Obst+verlust (Mädl III 1), ist in Knie- und Fersendistanz (Mädl I 8) die Verbindung von + distanz mit Körperteilen als Erstgliedern von Komposita ungewöhnlich.

Dies kann bei Nestroy als eine besondere Form der Realisierung einer Eigenschaft von Komödien angesehen werden, welche Fludernik (2015) bezeichnet als: "The comedy [...] resides in the clash of depicted elements, in the incongruity of the blended worlds". Zusammenfassend können wir feststellen, dass Nestroy offenbar sein Publikum durch einen überraschend geringen Grad an Kompatibilität der Glieder seiner okkasionalistischen Komposita beeindrucken wollte.

\section{Kotextuelle und kontextuelle Motivation von Nestroys Okkasionalismen}

Die syntagmatische Achse der Sprache ist über die Morphologie von Wörtern hinaus in der Einbettung von Okkasionalismen in den Kotext, d.h. in den syntaktischen und den übersatzmäßigen kohäsiven Zusammenhang und in den Situationskontext betroffen. Wie hier Nestroy seine Okkasionalismen kotextuell und kontextuell motiviert, ist noch nie systematisch untersucht worden.

Die kotextuelle Motivation erfolgt dabei sowohl eher durch den vorangehenden Kotext anaphorisch als durch den folgenden Kotext kataphorisch als auch umso eher, je kleiner der Abstand zwischen Okkasionalismus und dem motivierenden Kotext ist, was in semiotischer Perspektive zwei klaren indexikalischen Präferenzen folgt (Dressler 1989, 2000).

So fragt Lips (Zerriss II 9) die Magd Kathi, ob sie bereits jemanden umgebracht hat: „Na, wenn sich zum Beyspiel Einer aus Liebe zu dir was angethan hätt', wärst du seine indirecte Mörderin, Todgeber+in par distance“, wo der Okkasionalismus das durch die unmittelbar vorhergehende Nominalphrase motivierte okkasionalistische synthetische Kompositum präzisiert, was durch den Chiasmus indirecte $\mathrm{N}$ - N par distance noch zusätzlich verstärkt wird.

Anaphorisch motiviert ist auch der Okkasionalismus am Ende einer Reihe paralleler Wortbildungen in Mädl (II 7): „Hir die aimable Pfandlerinn, Wäschfabrikantinn und Hemdhandlerinn Mad[am] Storch, und hier ihre reitzenden Comi-innen." (Feminin-Motion aus Commis) Analog ist auch in Gewürz (I 15) Cichoris Ausführung über den ehemaligen Männerhass seiner jetzigen Frau: ,sie hat g'sagt, die Männer sind falsche Betrüger, Maulmacher, Bethörer, Sitzen-lasser".

Anaphorische Motivation findet sich in unmittelbar vorhergehenden Redebeiträgen in Mädl (III 1), wenn Madame Storch die bei Kauz eingeladenen Mädchen zurechtweist: „reißts nicht so viel Blumen $a b$ “. Rosalie meint darauf „Der [Madame Storch] ihre Lieblingsblumen sind die Blutzerbirn“. Sabine nimmt in ihrer Replik sowohl das Blumen- als auch das Obstmotiv durch eine okkasionalistische Gapping-Konstruktion auf und meint Herr Kauz werde den Blumen- und Obst-Verlust wohl leicht verschmerzen. 
Okkasionalismen können auch nur semantisch motiviert sein, wie in Gewürz (II 7), wo die beiden Gewürzkaufleute Baumöhl und Schwefel über die vermeintliche Untreue der Gattin des jeweils anderen diskutieren. Dabei komprimiert der Okkasionalismus Frauenentwendung die semantische Bedeutung der im Dialog vorangegangenen Worte stehlen, gestohlen, Diebstahl, Auf Schwefels „Einen andern Diebstahl zeigt man an“ erwidert Baumöhl: „Bey der Frauenentwendung blammirt man sich, wenn man ein G'schrey macht.“

Kataphorische statt anaphorischer Motivation ist viel seltener und funktioniert, wie gewöhnlich bei kataphorischen Bezügen (außer zwischen Titel und Text), nur auf kurze Distanz. So wird die Spanischerohr-Rache in Zerriss (I 4) im unmittelbar folgenden Satz als corsische Blutrache nachträglich motiviert.

Die „sprachliche Karikierkunst“ Nestroys (Zeman 2001: 179) zeigt sich häufig in durch den Situationskontext motivierten Okkasionalismen, die er bewusst einsetzt, um die satirische Wirkung von bestimmten Figuren oder Berufsgruppen zu betonen. Die Kombination von Okkasionalismen und der Wechsel von Sprachebenen dienen zudem der Verstärkung von Ironie und der Verfremdung (vgl. Hunger 1999: 24 ff.). Im Zerrissenen wird dieser Effekt beispielsweise durch die satirische Behandlung der Amts- bzw. Justizsprache erreicht. Der Justizbeamte, der für die Erbschaft des vermeintlich verstorbenen Herrn von Lips zuständig ist, tritt in der 12. Szene des 2. Aktes mit den Worten auf: „Nach nunmehro gepflogener Besichtigung des Schlosses, wolle es den verehrlichen pleno titulo Herren Erben des verewigten Herrn von Lips beliebsam seyn, zur Beaugenscheinigung der Pachthöfe zu schreiten." Der Okkasionalismus Be+augenschein+ig+ung verdichtet rechtliche Ausdrücke wie Berechtigung, Begnadigung, Bestätigung mit der Redewendung etwas in Augenschein nehmen. Lips bezichtigt er in der 7 . Szene des 3. Aktes der Schlossersäufungs-Inzicht und Lips müsse seine Unschuld am Tod des Schlossers Gluthammer beweisen, wobei er aber die „Absichtsverrathenden Worte des Testament-Wiederrufes“ in Betracht ziehen solle. Durch diese gekünstelte, übertrieben „geschraubte“ Sprache wird die mangelnde Authentizität der Figur unterstrichen und ihre negative Rolle innerhalb des Figurenpersonals betont. Nestroy setzt Okkasionalismen aber auch in einem größeren kontextuellen Rahmen ein, wenn er ebenfalls im Zerrissenen den übertriebenen Naturenthusiasmus der Romantik satirisch kommentiert. In Anspielung auf die im 19. Jahrhundert mit Hilfe des Verfahrens der Stereotypie hergestellten massenhaft produzierten Naturansichten beklagt sich Lips in I 6, dass die Natur ,auch an einer unerträglichen Stereotipigkeit“" erkrankt wäre und in II 9 möchte er sich gerne stundenlang in der „so einfältig angeverselten Landnatur" vergnügen. Die Wortneubildungen werden in beiden Kontexten mit einer bewusst negativen, abwertenden Intention eingesetzt und Kunstfertigkeiten wie Malen oder Dichten durch die Gleichsetzung mit handwerklichen einfachen Verrichtungen auf eine tiefere und abwertende Ebene transponiert.

\section{$7 \quad$ Vergleich von Nestroys Okkasionalismen mit denen seines Rivalen Berg}

Häufig wird die Bedeutung von Nestroys Wortwitz für das Überleben seiner Stücke auf der Bühne im Gegensatz zu den Volksstücken seiner Rivalen betont (vgl. Yates 1968). Können dabei auch Okkasionalismen eine Rolle gespielt haben? Bisher wurden Nestroys Okkasionalismen nie mit denen seiner Rivalen vergleichen. Das ermöglicht nun auch quantitativ das Viennese Theatre Corpus der ÖAW. Konkret haben wir Nestroys Okkasionalismen mit denen von vier Theaterstücken aus der Feder von Ottokar Franz Ebersberg (Pseudonym: O. F. Berg, geboren 1833, gestorben 1886): Ein Rekrut von 1859 (1859), Die alte Schachtel (1856), Der barmherzige Bruder (1874) und Wiener Karrikaturen (1880) verglichen. Es konnte festgestellt werden, dass Nestroy sowohl im Sinne der Quantität als auch in Hinblick der repräsentativen Verteilung der Wortbildungsmuster ein viel größerer Wortneubildner als sein Zeitgenosse war. In den insgesamt 93668 Worttoken der vier Stücke Bergs konnten nur 25 Okkasionalismen ausgemacht werden, somit etwas mehr als ein Viertel des Anteils von Okkasionalismen bei Nestroy. Lediglich drei Okkasionalismen überraschen durch ihre ungewöhnliche Beziehung zwischen den Kompositionsgliedern, darunter die Zehen+schweb+erei (Die alte Schachtel II 12). Generell ist zudem anzumerken, dass die spärlichen Okkasionalismen bei Berg wenig ko(n)textuelle Motivation aufweisen und vielmehr einer reinen zeitgeschichtlichen Satire und einer Steigerung einer platten Situationskomik geschuldet sind. Yates attestiert Nestroys Zeitgenossen generell ein Fehlen eines vergleichbaren Sprachbewusstseins und betont bei den anderen Komödiendichtern eine „Nachahmung von Nestroys geistvollem Spielen mit Worten“, welche zu einer „Reihe von leeren Kalauern“ (Yates 1968: 294) führte. 


\section{Welche Schauspieler sollten Nestroys Okkasionalismen sprechen?}

Eine solche Fragestellung haben wir noch in keiner einschlägigen Literatur gefunden. In Nestroys Theaterstücken sind nicht allein nur die Kompositionsart der Okkasionalismen und die Häufigkeit ihres Einsatzes im Vergleich zu den Texten seines Zeitgenossen auffallend. Aussagekräftig ist auch ihre Verteilung je nach Rolle. Nestroys Stücke waren stark mit dem sozialhistorischen Kontext des damaligen Theaterwesens verknüpft und mussten sich daher in die Praxis des tagesaktuellen Theatergeschäftes einfügen. Um ein langes Verbleiben am Spielplan und eine gute Einnahme zu garantieren wurden die Hauptrollen in den Stücken, so denn möglich, mit den zugkräftigsten Schauspielern der jeweiligen Bühne besetzt (vgl. Hein 1990: 47). So findet sich auch in den hier untersuchten Stücken eine durchwegs prominente Besetzungsliste vor: Die Hauptrollen Lips, Schnoferl und Zichori spielte Nestroy selbst, die Rolle von Gluthammer wurde von Wenzel Scholz verkörpert und Alois Grois spielte den Krautkopf und den Schwefel. Die Figur des Kauz im Mädl wurde vom Theaterdirektor des Theaters an der Wien, Carl Carl gespielt.

Bei einer näheren Analyse des Zerrissenen zeigt sich folgendes Zahlenbild. Lips, die männliche Hauptfigur, welche bei der Uraufführung am 9. April 1844 am Theater an der Wien von Nestroy selbst verkörpert wurde, spricht 44,7\% aller Okkasionalismen im Stück, kommt somit gemessen an der Anzahl seiner Okkasionalismen in Relation zu allen Wörtern auf einen Anteil von 0,00153\%, in Relation zu seiner Tokenanzahl auf einen Prozentsatz von 0,00037. Lips Gegenspieler Gluthammer (von Nestroys Freund Wenzel Scholz verkörpert) kommt rechnerisch auf 27,7\% der Okkasionalismen, damit anteilig gemessen an der Gesamttokenzahl auf 0,00087\%. In Relation zu seiner Tokenzahl aber auf 0,00071\%. Auf die weibliche Hauptperson Kathi entfallen lediglich 4,25\% der Okkasionalismen im Text, somit $0,00014 \%$, anteilig an ihrer Gesamttokenzahl 0,00011\%. Nestroy hat somit den Einsatz von Okkasionalismen strategisch geplant, um die theatralische Wirksamkeit zu erhöhen und den Hauptfiguren dadurch eine besonders große Sprach- und Ausdrucksfähigkeit zu verleihen. Sprachliche Fähigkeit beeinflusst somit maßgeblich die Stellung der Figur im Komödiengeschehen, je besser jene sind, desto wichtiger deren Sprecher.

Die (meist) männlichen Hauptfiguren, die sogenannten raisonneurs (Yates 1972: 96), die in der Regel von Nestroy selbst gespielt wurden, setzen gezielt Sprache, Kleidung und Mimik ein, um ihre Identität zu ändern. Die Sprache wird durch das Sprachbewusstsein dieser Figuren hinterfragt, sprachliche Äußerungen bloßgestellt und demaskiert. Die meist männlichen Hauptfiguren ziehen sich ein "G'wandl" an (Pape 2005: 28), die ihnen untergeordneten Rollen hingegen werden mithilfe einer zerfahrenen Sprache, einem bloßen stereotypen Nachplappern und dem Wiedergeben von nichtssagenden Plattitüden und Allgemeinplätzen als eher dumm und einfältig dargestellt.

\section{Peter Handke}

Peter Handke, geboren 1942 im zweisprachigen Kärntner Gebiet, betrat die Bühne des Literaturbetriebs mit seinen Erstlingen Die Hornissen (1966) und Publikumsbeschimpfung (1966) und genoss dort von Anfang an große Aufmerksamkeit, die seither nie wieder völlig aussetzte und in den 1990er Jahren rund um Handkes Reiseberichte aus dem zerfallenden Jugoslawien einen weiteren Höhepunkt erreichte. Seit der Jahrtausendwende widmet sich der Autor deutlich der Weiterentwicklung seines Projekts einer epischen Form, die sowohl seine Prosa als auch seine dramatischen Arbeiten bestimmt. Überhaupt lässt sich erkennen, dass Handke traditionelle Genres und die mit ihnen assoziierten Formen zunehmend umdeutet, wenn er etwa das bisher maßgeblich von Brecht definierte Oxymoron des epischen Theaters für sich neu bestimmt: „Episches Theater anders als in dem bekannten, zeigefingerkleinen, dogmatischen Sinn. Erzählerisches Theater, weiträumig, heimholend, märchenfremd und märchenvertraut, wie es mir mehr und mehr vorschwebt als das Theater" (Handke 2015: 21). Umgekehrt arbeitet der Autor in seinen großen Prosawerken mit Elementen des Dramatischen: „Der 'Bildverlust' ist ungeheuer dramatisch, man spürt: es geht um etwas; in diesem Sinne dramatisch. Es ist eine Dringlichkeit da; für mich ist die Dringlichkeit fast dramatischer oder leidenschaftlicher als ein sozusagen konstruiertes Drama." (Handke/Hamm 2008: 74)

Der dramatische Bildverlust (2002), ein laut Bezeichnung auf dem Umschlag der Taschenbuchausgabe als „Roman“ zu klassifizierender Text, der den Untertitel Durch die Sierra de Gredos trägt und als Fortsetzung der „Jugoslawien-Debatte“ mit epischen (oder eben dramatischen) Mitteln gelesen werden kann (vgl. Hafner 2008), ist auch der zentrale Untersuchungsgegenstand aus dem Werk Handkes in der 
vorliegenden Arbeit. Der Bildverlust bietet sich für eine literaturwissenschaftlich-linguistische Untersuchung deshalb an, weil er einerseits eine Vielzahl an poetischen Kernelementen des Handkeschen Oeuvres vereint, und andererseits aufgrund seines Umfangs eine große Textmenge zur linguistischen Analyse bietet. Als Vergleichsgröße wurde die kleinere Prosa Kali. Eine Vorwintergeschichte (2007) gewählt, die in relativer zeitlicher Nähe zum Bildverlust entstand und als Liebesgeschichte gelesen werden kann. Auch gattungstechnisch ist Kali ein interessanter Text, der gegen Ende mit einer bei Handke oft an Textschlüssen eingebrachten Gattungsthematisierung aufhorchen lässt: "Weg von den Dramen. Weg auch von den Liedern. Und auch genug gepredigt - wenn ihr andererseits dieses oder jenes Predigen hochhalten mögt. Zurück zur Prosa." (Kali 160) Schon Kastberger (2009: 155) hat auf die Besonderheit des Tons von Kali, der "teilweise hymnisch und selbst fast wie ein Gesang" ist, hingewiesen. Die beiden Texte bieten sich auch thematisch für einen Vergleich an. Beide Texte erzählen von weiblichen Hauptfiguren, wobei die Protagonistin von Kali eine Sängerin ist und jene des Bildverlusts "anfänglich selbst nicht weiß, dass sie eine Sängerin wird/ist und von der Handke-Rezeption bisher auch kaum als solche wahrgenommen wurde." (Özelt 2012: 295)

Die Untersuchung einzelner linguistischer Aspekte von Handkes Arbeit, etwa der Rolle von Konjunktionen (Federmair 2009) oder des Einsatzes der Wiederholung (Eisenhut 2008), wurde bereits von verschiedenen Forschenden geleistet - die okkasionalistische Wortbildung bei Handke ist bisher (außer in Hannesschläger \& Dressler 2017) noch nicht untersucht worden. Für die systematische Analyse dieses Phänomens wurde das Peter-Handke-Corpus der Academiae Corpora der ÖAW genutzt. Als Vergleichscorpus für die Erhebung der Geläufigkeit von Handkes Wortbildungen wurde Google, und damit das Internet als größtes Corpus der deutschen Gegenwartssprache, herangezogen.

\section{Handkes Okkasionalismen}

Wenn wir uns nun den okkasionalistischen Neubildungen einzelner Wörter zuwenden, so wird das Prinzip, dass ein Okkasionalismus nur ein einziges Mal bei einem Autor vorkommt, ein einziges Mal verletzt, nämlich im Bildverlust durch das Kompositum Stadtrand+idiot, welches immer ein und dieselbe Person bezeichnet, d.h. die Rekurrenz dieses Okkasionalismus ist durch die Rekurrenz der damit bezeichneten Person motiviert.

Handke bildet in seinen beiden Werken viele Okkasionalismen so gut wie immer nur nach produktiven Wortbildungsregeln, zum Unterschied von Nestroy keinen einzigen lexikalischen nach einem unproduktiven Muster. (Als unproduktiv könnte man höchstens die Komposition zweier Zeitadverbien in damals+jetzt [Bildverlust 100] bezeichnen.) Diese allgemeine Erkenntnis über die quasi ausnahmslos regelhafte, produktive Wortbildung in den untersuchten Texten kann direkt an Handkes Poetik rückgekoppelt werden: dieses Verfahren, mit dem Handke innerhalb der von der Sprache vorgeschlagenen Muster der Weltbeschreibung bleibt, entspricht seinem Projekt einer „neuen Klassik“ bzw. „ungewöhnlichen Klassik nach 1945“, wie Höller (2013) die Poetologie Handkes seit der rund um die Entstehung von Langsame Heimkehr (1979) diagnostizierten Wende im Schreiben des Autors mit einem pointierten Titel zusammenfasst.

Die beiden ungrammatischen Ausnahmen von den morphologischen Regeln des Deutschen sind Studentin+zimmer (ohne Interfix, Bildverlust 13) und Femininmotion des Suffixes -ling in Eindring +lin (Kali 100). So wie Nestroy hält sich also auch Handke an die Regeln der deutschen Grammatik und erlaubt sich fast gar keine darüber hinausgehende poetische Lizenz. Diese scheinbare sprachliche Nähe der beiden Autoren über Jahrhunderte hinweg sollte allerdings nicht automatisch als poetologische Nähe der beiden Autoren interpretiert werden; vielmehr liegt der Schluss nahe, dass eine regelhafte Verwendung des Deutschen unterschiedliche poetologische Zwecke erfüllen kann, und das über Jahrhunderte hinweg.

Handke hält sich auch (so wie Nestroy) an die Grundtendenz der kompositionsreichen deutschen Sprache, viel mehr neue Komposita als Derivationen zu bilden, angefangen mit dem Untertitel Vor+winter+geschichte (vgl. dazu weiter unten) von Kali. Er übertreibt sogar diese Tendenz, weil er nur ganz wenige okkasionalistische Derivationen kreiert, so die Konversion zickzack+ten (Bildverlust 68), das Partizip weg+geschmettert (Bildverlust 102), das deverbale Adjektiv taumel+ig (Bildverlust 118).

Der im Deutschen und darüber hinaus verbreiteten Präferenz, hauptsächlich zweigliedrige Komposita zu bilden, folgt auch Handke. Mehrgliedrige Komposita, besonders Phrasen- und Satzkomposita, 
sind durchaus selten, z.B. „Finanz+fürstin“+existenz (Bildverlust 22), Nie+wieder+zurückrufbaren (Bildverlust 10).

Zum Unterschied von Nestroy gehören die Elemente von Handkes Komposita großen Wortfamilien an, die wie alltägliche Neubildungen eine beträchtliche Familiengröße haben. So gibt es für den oben genannten Neologismus Vor+winter+geschichte eine große Familie von Wörtern, welche mit vor- beginnen, Winter- ist ein häufiges Erstglied und -geschichte ein häufiges Zweitglied. Hingegen kann man bei diesem Kompositum nicht leicht Haupt- und Nebengrenze unterscheiden, d.h. sich für rechtsverzweigendes Vor+wintergeschichte oder für linksverzweigendes Vorwinter+geschichte entscheiden.

Wie in all diesen Eigenschaften folgt Handke der Alltagssprache und der klassischen Literatur in der intertextuellen Motivation von Okkasionalismen, so durch anklingende und analog gebaute existierende Wortbildungen (vgl. dazu allgemein Mattiello 2016), z.B. in ihrer erd+umspinnenden Legende (Bildverlust 16: in Analogie zu erd+umspannend).

Noch klarer ist die Motivation durch den Kotext, wie zu erwarten, besonders durch den vorangegangenen und bei geringer Distanz zwischen motivierenden Elementen und dadurch motiviertem Okkasionalismus, wie in „Dann folgt das Kind ihrem Blick [...] Könnte auch ich so ihrem Blick folgen? Ist das jetzt ein beiderseitiger Kom-plizen+blick?" (Kali 18).

Dies kann auch zu Parallelismen von Okkasionalismen führen bzw. zum Parallelismus eines Okkasionalismus mit einem bereits verwendeten Kompositum, wie in Die zauberische Montags+leere, Wochenanfangs+leere (Bildverlust 64). Hier zeigt sich, dass Handkes Okkasionalismen immer aus dem Versuch entstehen, einen präzisen Begriff für ein vom Autor in der Welt beobachtetes Phänomen zu finden. Das gilt für die Okkasionalismen bei Handke allgemein und zeigt sich in diesem Beispiel in mehrfacher Form: Handke nutzt den (zwar selten, aber doch auch andernorts nachgewiesenen - 62 Beispiele liefert Google) Begriff der Montagsleere und präzisiert das Gemeinte, indem er es auf eine allgemeinere Ebene hebt, als Wochenanfangsleere. Damit geht Handke vom engeren zum breiteren, vom etablierten zum neu geschaffenen Begriff, um das Besondere im Allgemeinen sichtbar zu machen und einen allgemeinen Begriff für ein besonderes Phänomen erst in die Sprache einzuführen, und zwar mit ihren eigenen Mitteln. Ein besonders eindrückliches Beispiel eines aus dem Thema des Texts direkt erwachsenden Okkasionalismus, der im Zuge des Versuchs einer besonders präzisen Verwortung eines Phänomens entsteht, ist die in Kali genannte Salzofen+stille (127).

Die hier behandelten Beispiele zeigen, dass Handke (anders als Nestroy) nicht oder nur sehr selten zur effekt- und humorvollen Pointe neigt, dass er vor allem mit der Bildung von Okkasionalismen nicht auf diese abzielt, sondern sie dort kreiert, wo das richtige Wort zur Weltwiedergabe fehlt. Das bedeutet nicht, dass Handkes Literatur im Allgemeinen humor- oder pointenfrei wäre - im Gegensatz zu Nestroy werden diese rhetorischen Instrumente bei Handke allerdings sparsamer eingesetzt. Ein Beispiel ist die Gattungsbeschreibung, die am Ende des Bildverlusts mit Reim, Kalauer und Sprichwort gleichzeitig operiert: "Und damit war die Geschichte aus, und wir gingen nachhaus. Im Erzähllokal wurden die Lichter abgedreht." (Bildverlust 759)

\section{Gapping}

Wodurch sich Handke in der okkasionalistischen Wortbildung auszeichnet, ist seine Vorliebe für die aus einer Interaktion von Syntax und Wortbildung bestehende Gapping-Konstruktion (Kompositumskoordinationskürzung), besonders im Bildverlust (350 Beispiele; in Kali - einem allerdings auch wesentlich kürzeren Text - nur 30). Gapping (Booij 1985, Wälchli 2015), wie im Grundtyp in der Form Haupt- und Staats+zeiten (Bildverlust 27) besteht in der Verbindung zweier (oder viel seltener mehrerer) gleichgebauter Wortbildungen, bei denen die gleichbleibende Konstituente zunächst weggelassen und nur beim letzten komplexen Wort beibehalten wird. Wie in diesem Beispiel erfolgt die Kürzung fast immer nur im Vorgängerkompositum, nicht im letzten Kompositum der Gappingkonstruktion, was dem allgemeinen Sprachgebrauch entspricht. Denn bei der Sprachverarbeitung ist das in Komposita rechts befindliche Haupt des Kompositums leichter rekonstruierbar als das links befindliche Nichthaupt, wie in hierher+gewanderten und -geflüchteten (Bildverlust 37), und weil in mündlicher Sprache der Bindestrich nach dem Erstglied (aber nicht der Bindestrich vor dem Zweitglied) eine prosodische Entsprechung hat. Wie in der Alltagssprache kommen bei Handke Gappingkonstruktionen von Derivationen kaum vor, z.B. vater- und mutter+loses Kind (Kali 59). 
Es lässt sich aus dieser häufigen, aber geläufigen Verwendung von Gapping bei Handke schließen, dass er dieses linguistische Phänomen für sich entdeckt hat und dort zum Einsatz bringt, wo die Dinge in der Schwebe bleiben, wo sie gleichberechtigt das eine und das andere (oder das eine oder das andere) sind oder sein könnten, wo zwei Elemente eines Spektrums gleichzeitig der Fall sind (oder sein könnten). Dabei geht es dem Autor aber darum, das Publikum auf die jeweiligen Elemente aufmerksam zu machen und es für ihre Wahrnehmung zu öffnen, und nicht darum, durch ungeläufige oder gar unregelhafte Sprachverwendung (also Struktur) vom Thema (also Inhalt) abzulenken.

Nahezu 99\% der Gappingkonstruktionen sind okkasionalistisch. Die gängigen und/oder mehr als einmal verwendeten Okkasionalismen, die also nicht für eine einzige Textstelle erfunden wurden, beziehen sich hingegen oft auf beliebte Themen Handkes: Beispiele dafür sind etwa das Ein- und Ausatmen (Bildverlust 83, 720), das Kreuz- und Quergehen (Bildverlust 569, 658) oder das Vater- und Mutterland (Bildverlust 599), das „der Autor“ verlassen hat. Letzteres Beispiel kommt im Handke-Corpus ein weiteres Mal vor, im Jugoslawien-Stück Die Fahrt im Einbaum oder Das Stück zum Film vom Krieg (1999: 51) und steht damit bei Handke deutlich im Kontext der Frage nach Zugehörigkeit und eines Konzepts von Heimat, die sich aus (Familien-)Herkunft ableitet. Dieses Motiv ist bei Handke ein geläufiges, wenngleich in den meisten Texten, in denen Heimat und Herkunft thematisiert werden, diese über eine mütterliche Familie verhandelt werden (etwa in Die Wiederholung und Immer noch Sturm; vgl. Hannesschläger 2017).

Wenn zusätzlich auch eines der Komposita der Gappingkonstruktion selbst ein Okkasionalismus ist, dann steht dieser fast immer an zweiter Stelle, wie in den schon erwähnten Haupt- und Staats+zeiten. Dies entspricht einem natürlichen Fortschritt vom Bekannten zum Neuen und ist auch leichter zu verarbeiten, wenn, wie meist, das letzte (also okkasionalistische) Kompositum ungekürzt ist und nur ein bereits bekanntes Kompositum gekürzt wird. Wie in der Alltagssprache gibt es daher kein Gapping in der Aufzählung Eine Kochmütze. Ein Kochhalstuch. Eine Kochschürze. Ein Kochknieschutz. Kochpantoffeln aus Lindenholz (Bildverlust 126). Anders als in den vorangegangenen Beispielen mit Gapping, wo Handke Parallelitäten, Gleichzeitigkeiten und die Schwebe zwischen den mittels Gapping verbundenen Elementen in den Vordergrund stellt, zeigt dieses Aufzählungsbeispiel, dass die Besonderheit der einzelnen Elemente hervorgehoben werden soll, die jeweils für sich stehen. Jedes dieser Kochutensilien verlangt für sich Aufmerksamkeit, könnte auch alleine stehen. Dagegen können mittels Gapping ebensoviele Elemente nebeneinandergestellt werden, deren individuelle Besonderheit erst durch die Verbindungen zwischen ihren Einzelaspekten deutlich wird; das ist etwa der Fall, wenn die einstigen Obst- und Gemüse + gärten oder -felder oder -terrassen (Bildverlust 48) durch eine komplexe Gappingkonstruktion hervorgehoben werden, deren potentielle Vielgestaltigkeit ihre wesentliche Eigenschaft darstellt.

\section{Charakteristika der Koordination innerhalb der Gappingkonstruktionen}

Die Koordination ist bei Handke gewöhnlich, wie in der Alltagssprache, syntaktisch sehr eng, d.h. die Verbindung erfolgt meist durch eine einzige einsilbige Konjunktion, besonders und (276 Bespiele im Bildverlust), oder (110), bis, wie usw. Mehrere Elemente, welche viel seltener zur Koordination verwendet werden sind oder eher, oder auch, oder schon, und vor allem, sowie, für die Alltagssprache ungewöhnlich, und (beinahe) auch usw. Ganz selten ist Asyndese, wie in Die Winter-, die Januar+Reisende (Bildverlust 80). Die Vermeidung von Asyndese passt zu Handkes allgemeiner Tendenz der besonders engen Verbindung der koordinierten Komposita. Der Enge der Verbindung dient auch die Vermeidung von Artikeln innerhalb der Gappingkonstruktionen. Das interne Fehlen der ziemlich inhaltsleeren Artikel lenkt die Aufmerksamkeit noch stärker auf die eingesetzten Begriffe, also die thematisierten Elemente.

Die syntaktisch enge Koordination suggeriert ikonisch eine semantisch enge Verbindung der koordinierten Komposita, welche aber meist in der Realität nicht besteht, sondern nur in der vorgestellten Textwelt Peter Handkes. So handelt es sich beim häufigsten Typ, der bloßen Verbindung durch und, dass es sich nie um semantisch enge Verbindungen des Typs Augen und Ohren, sondern die Konstruktion referiert einfach auf nebeneinander koexistierende Entitäten, die der Autor auf den ersten Blick willkürlich zusammenstellt. Dabei zeigt sich aber bei genauerer Analyse, dass er damit die Lesenden auf Zusammenhänge zwischen Begriffen aufmerksam machen möchte, die sich nicht unmittelbar erschließen, sich aber durch die Verbindung mit dem gemeinsamen Zweitglied eröffnen, wie in Geld- und Computer+diktatur (Bildverlust 96) oder in die Traum- und Geister+erählungen in Tibet (Bildverlust 
37). Diese enge Verbindung einer Traumwelt mit Geistergestalten (also Toten) darf als einer der wesentlichen Motoren des Handkeschen Schreibens bezeichnet werden; über Immer noch Sturm, ein an den Biographien der toten Vorfahren mütterlicherseits entlang entwickeltes Drama (vgl. Hannesschläger 2017), sagte der Autor etwa, er habe "fünfzehn Jahre herumgeträumt an diesem Stück" (Handke/Patterer/ Winkler 2012: 30, unsere Hervorhebung).

Die Verbindung mit dem gemeinsamen Zweitglied kann auch eine Intensivierung oder Präzisierung mit sich bringen, wie in das Bedeutungslos- und Unscheinbar+werden der Vorfahren (Bildverlust 11). Die Konjunktion und erweist sich also besonders in Handkes Gappingkonstruktionen als additives Verbindungswort, das auf die Koexistenz der verbundenen Elemente hinweist und die Pluralität der Erscheinungsformen der Welt betont.

Die in Gappingskonstruktionen am zweithäufigsten verwendete Konjunktion oder hat in okkasionalistischen Konstruktionen so gut wie nie eine exklusive Bedeutung (Lat. aut), d.h. im Sinne von entweder $X$ oder $Y$ (Lat. aut - aut). Dazu passt, dass entweder $X$ oder $Y$, welches Handke ansonsten relativ häufig verwendet, bei Gapping in den beiden untersuchten Texten überhaupt nur ein einziges Mal vorkommt, nämlich in ihr das Schlechtestmögliche entweder nach- oder vorherzusagen (Bildverlust 61).

Sonst verwendet Handke in Gappingkonstruktionen oder im Sinne von oder auch (Lat. vel), d.h. ähnlich wie und, etwas in so sah sie eines Sommer-oder Wintermorgens (Bildverlust 10) oder in die fernen alpen- oder pyrenäen+hohen Bergketten (Kali 34). Dazu kann der zweite Ausdruck den ersten zumindest leicht verbessern, etwa in als eine Art Asyl-oder Ausruhzone (Bildverlust 31), was an die Verwendung von oder eher erinnert, etwa in auf die Gegen- oder eher Nebenspur (Bildverlust 15). Daher bedeutet oder in Gappingkonstruktionen nie eine vollständige, eindeutig exkludierende Korrektur, weshalb wir in In meiner Kindergegend. Oder nein, in der Nachbargegend (Kali 30) auch kein Gapping finden. Die einzige Ausnahme bildet keine Liebes-, sondern eine Kampfnacht (Bildverlust 165). Die fast ausschließlich additive Verwendung von oder in Gappingkonstruktionen stützt somit weiter unsere oben formulierte These, dass Gapping bei Handke vor allem dort zum Einsatz kommt, wo die Schwebe zwischen gleichberechtigten Phänomenen der Welt zum eigentlichen Thema des Schreibens wird.

Diese These erklärt auch Handkes Vermeidung von Negationen. Analog zur Vermeidung von entweder-oder kommt weder-noch bei Gapping gar nicht vor, obwohl wir sonst im Bildverlust 79 Beispiele von weder-noch vorfinden. Besonders stark vermeidet Handke eine negative Verbindung innerhalb der Gappingkonstruktion, wohl aber in übergeordneter Funktion, also außerhalb der Gappingkonstruktion: keine Gemeinschafts- und schon gar keine Gesellschafts+gefühle mehr (Bildverlust 89), kein Anhauch von Todes- oder Vergehens+angst (Bildverlust 222). Diese Beispiele zeigen, dass Handkes zentrales Anliegen die Betonung von Gleichzeitigkeit und Vielschichtigkeit von Phänomenen selbst dann ist, wenn er diese Phänomene im Rahmen einer gemeinsamen Negation ausschließt.

\section{Okkasionalismen bei Handke und Nestroy: (k)ein Vergleich}

Die sprachliche Kreativität Handkes lässt sich anhand der angeführten Beispiele von Okkasionalismenbildung und Einsatz von Gappingkonstruktionen so zusammenfassen: Der Autor drückt mit normalen, erlaubten Mitteln die Schönheit und Spezifizität der Welt aus und beschreibt diese immer am Traum entlang entstehende Welt, die er erst aus der Sprache schafft, mit den Mitteln, die diese ihm anbietet. Die beiden untersuchten Texte fügen sich damit auch aus linguistischer Perspektive in die diagnostizierte Handke'sche „Wende zum Klassischen“ (Höller 2013). Dabei konnten wir in allen untersuchten Beispielen immer eine klare inhaltliche Motivation für den Einsatz von Okkasionalismen und Gapping finden; im Gegensatz zu Nestroy greift Handke auf diese sprachlichen Mittel dann zurück, wenn "es ernst wird", also dann, wenn ein präziser Ausdruck für die Beschreibung eines Phänomens notwendig ist. Bei Handke ohnehin nur sehr sparsam gesetzte Pointen werden nicht mit diesen sprachlichen Mitteln gebaut.

Nestroy setzt in seinen Theatertexten Mimik, Gestik und vor allem das Spiel mit der Sprache, gleich ob es sich hierbei um den gekonnten Wechsel verschiedener Sprachebenen (Hochsprache, Umgangssprache, Dialekt), dem Einsatz von Metaphern, Vergleichen oder kontextuell bedingten Verwendung von Okkasionalismen handelt, gezielt ein, um die theatralische Wirkung des Wortwitzes zu steigern (vgl. Hüttner 2005). Die Okkasionalismen werden als Klimax in den dialogischen und monologischen Strukturen der Texte positioniert, erhöhen damit die Aufmerksamkeit des Publikums. Sie werden in den meisten Fällen von den raisonneurs gesprochen und signalisieren deren Bereitschaft der Reflexion über 
die Sprache und ermöglichen diesen Figuren in (selbst)kritischer Distanz zu ihrer Funktion innerhalb des Handlungsablaufes zu treten. Sprache wird hiermit als Mittel zur Änderung der Identität einer Figur eingesetzt, erzeugt doppelte Distanz und offenbart die „maskenhafte Verstellungskunst der nestroyschen komischen Hauptfiguren“ (Pape 2005: 24). Der Definition von Posner folgend, sind Nestroys Theatertexte ,poetisch lebendig“ (Posner 1973: 518). Ihr Sprachwitz und ihre Sprachkomik garantieren die dauerhafte Präsenz von Nestroys Werken auch in der Gegenwart, Nestroys Okkasionalismen sind demnach ein wichtiges Kriterium für die Literarizität seiner Theatertexte.

\section{Schlussbemerkung}

In dieser interdisziplinären Zusammenarbeit konnten wir zeigen, wie mit linguistischen, besonders korpuslinguistischen Mitteln die poetische Kreativität, exemplarisch dargestellt anhand eines Dramatikers und eines Epikers der österreichischen Literatur, näher bestimmt werden kann. Für die Literatur- (und, bei Nestroy, auch Theater-)Wissenschaft ist diese Untersuchungsmethode für neue Erkenntnisse fruchtbringend und ermöglicht eine tiefgehendere, strukturiertere Auseinandersetzung mit den analysierten Texten. Korpuslinguistisches Arbeiten mit dramatischen Texten erlaubt eine entschleunigte, fokussiertere Konzentration auf den Text, während epische Texte mit punktuellem Fokus auf einzelne Textstellen anstelle von Konzentration auf den darin verhandelten großen thematischen Bogen gelesen werden können. Für die Linguistik bietet sich die Möglichkeit zu untersuchen, wie Literaten die Potentialität einer Sprache kreativ ausschöpfen und damit über alltägliche sprachliche Neubildungen hinausgehen.

\section{Literatur}

\section{Primärliteratur}

Ottokar Franz Ebersberg. 1860. Ein Rekrut von 1859. Volksstück mit Gesang in 3 Abtheilungen. Wallishausser, Wien. (=Wiener Theater-Repertoir 54).

Ottokar Franz Ebersberg. 1865. Die alte Schachtel. Lebensbild mit Gesang in 3 Akten. Schweiger \& Comp., Wien.

Ottokar Franz Ebersberg. 1880. Wiener Karrikaturen. Posse in Gesang in 5 Bildern. Wien, Selbstverlag. Ottokar Franz Ebersberg. 1876. Der barmherzige Bruder. Posse mit Gesang in 7 Bildern. Wallishausser, Wien (=Wiener Theater-Repertoir 310).

Peter Handke. 1999. Die Wiederholung. Suhrkamp, Frankfurt am Main.

Peter Handke. 2003. Der Bildverlust oder Durch die Sierra de Gredos. Frankfurt am Main, Suhrkamp (=suhrkamp taschenbuch 3519).

Peter Handke. 2008. Kali. Eine Vorwintergeschichte. Suhrkamp, Frankfurt am Main, (=suhrkamp taschenbuch 3980).

Peter Handke. 2010. Immer noch Sturm. Suhrkamp, Berlin.

Peter Handke. 2015. Tage und Werke. Begleitschreiben. Suhrkamp, Berlin.

Peter Handke und Peter Hamm. 2006. Es leben die Illusionen. Gespräche in Chaville und anderswo. Wallstein, Göttingen. 2. Auflage 2008.

Peter Handke im Gespräch mit Hubert Patterer und Stefan Winkler. 2012. Edition Kleine Zeitung, Graz. Johann Nestroy. 1977-2004. Sämtliche Werke. Historisch-kritische Ausgabe. Hg. von J. Hein, J. Hüttner, W. Obermaier und W. E. Yates. Deuticke, Wien. [HKA].

\section{Sekundärliteratur}

Norbert Bachleitner. 1989. Übersetzungsfabriken. Das deutsche Übersetzungswesen in der ersten Hälfte des 19. Jahrhunderts'. Internationales Archiv für Sozialgeschichte der deutschen Literatur 14(1). $1-49$.

Geert Booij. 1985. Coordination reduction in complex words: a case for prosodic phonology. In H. van der Hulst und N. Smith (Hg.). Advances in Non-linear Phonology. Dordrecht, Foris. 143-168.

Erik Iossifovič Chanpira. 1966. Ob okkazional'nom slove i okkazional'nom slovoobrazovanii. In E.A. Zemskaja und D.N. Šmelev (Hg.). Razvitie slovoobrazovanija sovremennogo russkovo jazyka. Nauka, Moskau. 153-166.

Anastasia Christofidou. 1994. Okkasionalismen in poetischen Texten: Eine Fallstudie am Werk von O. Elytis. Narr, Tübingen. 
Susan Doering. 1994. Es ist alles uralt, nur in andrer Gestalt: Über die Vorlagen von Nestroys Werken. In W. E. Yates (Hg.). Vom schaffenden zum edierten Nestroy. Jugend \& Volk, Wien. 31-44.

Wolfgang U. Dressler. 1981. General principles of poetic license in word formation. In H. Geckeler et al. (Hg.). Logos Semantikos, Fs. E. Coseriu II. de Gruyter, Berlin \& Gredos, Madrid. 423-431.

Wolfgang U. Dressler. 1989. Semiotische Parameter einer textlinguistischen Natürlichkeitstheorie. Verlag der Österreichischen Akademie der Wissenschaften, Wien.

Wolfgang U. Dressler. 2000. Textlinguistik und Semiotik. In K. Brinker et al. (Hg.). Text- und Gesprächslinguistik. I. de Gruyter, Berlin. 762-772.

Wolfgang U. Dressler. 2007. Produktivität und poetische Lizenz. In W. U. Dressler und O. Panagl (Hg.). Poetische Lizenzen. Praesens Verlag, Wien. 117-132.

Wolfgang U. Dressler und Karlheinz Mörth. 2012. Produktive und weniger produktive Komposition in ihrer Rolle im Text an Hand der Beziehungen zwischen Titel und Text. In L. Gaeta und B. Schlücker (Hg.). Das Deutsche als kompositionsfreudige Sprache. de Gruyter, Berlin. 219-233.

Johannes J. Eisenhut. 2008. Wieder holen. Kognitive Linguistik in der Literaturwissenschaft. Exemplarische Untersuchungen zur Wiederholung in Texten von Peter Handke. Saarbrücken, VDM.

Leopold Federmair. 2009. Formen der Konjunktion: Zum Verhältnis von Chronik und Epos bei Peter Handke. In K. Kastberger (Hg.). Peter Handke: Freiheit des Schreibens - Ordnung der Schrift. Paul Zsolnay, Wien, 306-324.

Ernst Fischer. 1962. Von Grillparzer zu Kafka: sechs Essays. Globus Verlag, Wien.

Monika Fludernik. 2015. Blending in cartoons: the production of comedy. In L. Zunshine (Hg.). The Oxford Handbook of Cognitive Literary Studies. Oxford University Press, Oxford. 155-175.

Livio Gaeta und Beate Schlücker (Hg.). 2012. Das Deutsche als kompositionsfreudige Sprache. de Gruyter, Berlin.

Dagmar Gengnagel. 1962. Zur sprachlichen Gestaltung der Possen Johann Nestroys. In Wissenschaftliche Zeitschrift der Friedrich-Schiller-Universität Jena 11(1). 119-130.

Fabjan Hafner. 2008. Peter Handke. Unterwegs ins Neunte Land. Paul Zsolnay, Wien.

Vanessa Hannesschläger. 2017. Real Life Fiction, Historical Form: Peter Handke's 'Storm Still'. In L. Boldrini, und J. Novak (Hg.). Experiments in Life-Writing. Intersections of Auto/Biography and Fiction [Palgrave studies in Life-writing 1]. Palgrave, London. 145-165.

Vanessa Hannesschläger und Wolfgang U. Dressler. 2017. Poetische Brücken über sprachliche Lücken. Kompositabildung und Gapping in Peter Handkes "Bildverlust" und "Kali" analysiert mit corpuslinguistischen Methoden. In Studia austriaca 25 (2017). 119-140.

Jennifer Hay und Harald Baayen. 2002. Parsing and productivity. Yearbook of Morphology 2001. 203235.

Jürgen Hein. 1970. Spiel und Satire in der Komödie Johann Nestroys. Koch Buchverlag GmbH, Bad Homburg, Berlin, Zürich.

Jürgen Hein. 1990. Johann Nestroy. Metzler, Stuttgart.

Hans Höller. 2013. Eine ungewöhnliche Klassik nach 1945. Das Werk Peter Handkes. Suhrkamp, Berlin.

Herbert Hunger. 1999. Das Denken am Leitseil der Sprache: Johann Nestroys geniale wie auch banale Verfremdungen durch Neologismen. Verlag der Österreichischen Akademie der Wissenschaften, Wien.

Johann Hüttner. 2005. Spiele mit Worten. Zum Unverständnis von Nestroys Komik. In H. Haider-Pregler et al. (Hg.). Komik. Ästhetik. Theorien. Strategien. [=Maske und Kothurn. Internationale Beiträge zur Theater-, Film- und Medienwissenschaft. 51 (4)]. Wien, Köln, Weimar. 159-167.

Klaus Kastberger. 2009. Peter Handke und das Salz - fünf Orte. In K. Kastberger (Hg.). Peter Handke. Freiheit des Schreibens - Ordnung der Schrift [= Profile. Magazin des Österreichischen Literaturarchivs der Österreichischen Nationalbibliothek (16)]. Paul Zsolnay, Wien. 143-156, 174-182.

Karl Kraus. 1912. Nestroy und die Nachwelt. Zum 50. Todestage. In Die Fackel 349/350 vom 13. Mai 1912.

Lothar Lemnitzer und Heike Zinsmeister. 2006. Korpuslinguistik. Eine Einführung. Gunter Narr, Tübingen.

Anke Lüdeling und Amir Zeldes. 2009. Three Views on Corpora: Corpus Linguistics, Literary Computing, and Computational Linguistics. In Jahrbuch für Computerphilologie (9). 149-178.

Elisa Mattiello. 2016. Analogy in New English Words. de Gruyter, Berlin. 
Kimberley Mulder, Ton Dijkstra, Robert Schreuder und R. Harald Baayen. 2014. Effects of primary and secondary morphological family size in monolingual and bilingual word processing. In Journal of Memory and Language 72. 59-84.

Judith Murat. 2015. Lexical Creativity. In R. H. Jones (Hg.). The Routledge Handbook of Language and Creativity. Routledge, London.

Clemens Özelt. 2012. Klangräume bei Peter Handke. Versuch einer polyperspektivischen Motivforschung. Braumüller, Wien.

Oswald Panagl. 1983. (De-) Lexikalisierung und Paretymologie im Wortschatz Johann Nestroys. In M. Dardano (Hg.). Parallela, Akten des 2. österreichisch-italienischen Linguistentreffens. Tübingen. 64-79.

Oswald Panagl. 2003. Im Anfang war das Wort. In W. Berka, E. Brix und C. Schmekal (Hg.). Woher kommt das Neue? Kreativität in Wissenschaft und Kunst [=Wissenschaft Bildung Politik Band 6]. Böhlau, Wien-Köln-Weimar. 149-169.

Walter Pape. 2005. „Da heißt's jeder Red“ a Fey'rtagswand'l anzieh'n“: Sprache und Gebärde, Verstellung und Verkleidung in Nestroys Komödien. In Nestroyana. Blätter der Internationalen NestroyGesellschaft 25 (1/2). 16-30.

Charles William Peppler. 1902. Comic Terminations in Aristophanes and the Comic Fragments: Diminutives, Character Names, Patronymics. John Murphy Co, Baltimore.

Roland Posner. 1973. Linguistische Poetik. In Lexikon der germanistischen Linguistik. Niemayer, Tübingen. 513-522.

Hans-Georg Ruprecht. 1965. Eugène Scribes Theaterstücke auf den Bühnen in Wien, Leipzig, Weimar und Berlin. Eine geschmackssoziologische Studie über den Erfolg der Scribschen Theaterstücke in den deutschsprachigen Bearbeitungen des 19. Jahrhunderts. Diss. Saarbrücken.

Friedrich Sengle. 1980. Biedermeierzeit. Deutsche Literatur im Spannungsfeld zwischen Restauration und Revolution 1815-1848, Band III: Die Dichter. Metzler, Stuttgart.

Angelika Storrer. 2011. Korpusgestützte Sprachanalyse in Lexikographie und Phraseologie. In K. Knapp et al. (Hg.). Angewandte Linguistik. Ein Lehrbuch. Franke Verlag, Tübingen. 3. Auflage. 216-140.

Barbara Tumfart. 2012. Eugène Scribes Theater im Taumel der Gefühle. Emotionen in deutschsprachigen Übersetzungen des 19. Jahrhunderts. In Nestroyana. Blätter der Internationalen Nestroy-Gesellschaft 32 (3/4). 146-162.

Barbara Tumfart. 2018. Über „das dramatische Waarenlager des regsamen Importeurs“ Alexander Bergen alias Marie Gordon (1812-1863). Auf den Spuren einer Dramenübersetzerin des 19. Jahrhunderts. In Nestroyana. Blätter der Internationalen Nestroy-Gesellschaft 38 (1/2). 56-68.

Bernhard Wälchli. 2015. Co-compounds. In P. Müller et al. (Hg.). Word-Formation. An International Handbook of the Languages of Europe. Mouton de Gruyter, Berlin. 707-727.

Walter Weiss. 1995. Zur Tradition der Thematisierung der Sprache in der österreichischen Literatur. Sprachskepsis und Sprachmagie bei Georg Trakl und Peter Handke. In W. Weiss (Hg.). Annäherungen an die Literaturwissenschaft. I. Literatur-Sprache. Heinz, Stuttgart. 199-210.

William E. Yates. 1972. Nestroy: Satire and Parody in Viennese Popular Comedy. Cambridge Univ. Press, Cambridge.

William E. Yates. 1968. Nestroysche Stilelemente bei Anzengruber. Ein Beitrag zur Wirkungsgeschichte der Possen Nestroys. In Maske und Kothurn. Vierteljahrsschrift für Theaterwissenschaft 14. 287-296.

William E. Yates. 2008. Continuity and Discontinuity in Viennese Theatrical Life from the 1860 s to the Turn of the Century. In Austrian Studies 16. 51-68.

Herbert Zeman. 2001. Johann Nepomuk Nestroy. Holzhausen, Wien. 